\title{
Application of the finite element method for static design of plane linear systems with semi-rigid connections
}

\author{
D. Zlatkov, S. Zdravkovic, B. Mladenovic \& M. Mijalkovic \\ Civil Engineering and Architectural Faculty of Nis, Serbia
}

\begin{abstract}
In real constructions, particularly in prefabricated constructions, structural connections can be neither absolutely rigid nor ideally elastic, but semi-rigid, which significantly changes the stresses and strains in the structure. Hence, there is a need to carry out the structural analysis and design taking into account the level of rigidity of the connections. For that purpose the ratio between real and absolutely rigid fixing of the member ends is assumed to be from zero to one.

The design procedure for structures with semi-rigid connections under static load based on the classical deformation method has already been described in our previous works. Having in mind that matrix formulation of a problem is more convenient for contemporary structural analysis, this is applied to the design of the considered systems and is described in detail in this paper.

The formation of the stiffness matrix for a bar with semi-rigid connections, as well as the vector of the equivalent load, is shown to depend on the level of rigidity of a joint connection. These matrices can be introduced into well-known computer programs to modify them for the static design of a plane linear system whose connections are semi-rigid.

A numerical example regarding a two-floor reinforce concrete frame with a span of $24 \mathrm{~m}$ of the AMONT prefabricated structural system in Morava Krusce, Serbia, is included in the paper.

Keywords: semi-rigid connections, stiffness matrix, interpolation function, vector of equivalent load, prefabricated reinforced concrete structural system.
\end{abstract}




\section{Introduction}

Many worldwide researches, based on numerical simulations and experimental results, indicated that a great number of connections of members in joints of linear systems can neither be classified as ideally pinned nor as absolutely rigid. The results of an integral project devoted to attesting the static and dynamic stability of typified modules of the reinforced concrete (RC) AMONT structural system of industrial halls realized in IZIIS, Skopje, Macedonia (Ristic et al. [5]), together with the Institute of Civil Engineering and Architectural Faculty at Nis, confirmed this statement. It has been noticed from the tests performed that the level of rigidity of connections is of great importance, particularly in the case of precast structures, because even a low level of rigidity in precast connections affects the redistribution of action effects.

With the purpose of including the real rigidity of connections in the structural design (Milicevic et al. [2]), the ratios between real and absolutely rigid the fixing of the member ends are assumed to be $\mu_{i k}$ and $\mu_{k i} \quad\left(0 \leq \mu_{i k} ; \mu_{k i} \leq 1\right)$, where:

$$
\mu_{i k}=\varphi_{i k}^{*} / \varphi_{i}, \mu_{k i}=\varphi_{k i}^{*} / \varphi_{k}
$$

With this assumption about the level of rigidity, the design procedure for structures with semi-rigid connections is developed using the deformation method. The classical formulation of the deformation method for systems with standard connections has already been described (Djuric and Jovanovic [1]), as well as for systems with semi-rigid connections (Milicevic and Zdravkovic [2]). In this paper, the deformation method is applied in matrix formulation taking into account assumption (1) and the derivation of the stiffness matrix, and the vector of the equivalent load has been carried out using the variational procedure.

\section{Matrix analysis of systems with semi-rigid connections}

Matrix analysis can be considered as a special case of the finite element method, a well known method of numerical structure analysis. In the matrix formulation of the force method and the deformation method, the basis is a member as one dimensional finite element. The system is discrete, composed of memberselements of the system, which are interconnected in the discrete points (joints of a system).

Fig. 1 shows the simplest model of a straight prismatic member of length $l$, of a constant cross section area, exposed to bending in the plane xoy of the local coordinate system. The member cross section moment of inertia is I and the material modulus of elasticity is E. If the influence of axial forces on the deformation of the member is neglected, the generalized displacements are transversal displacements $\left(\boldsymbol{v}_{\mathrm{i}}, \boldsymbol{v}_{\mathrm{k}}\right)$ and rotations $\left(\boldsymbol{\varphi}_{\mathrm{i}}, \boldsymbol{\varphi}_{\mathrm{k}}\right)$ of the member ends, thus the element has four degrees of freedom, two at each joint. Generalized 
forces are shear forces $\left(\boldsymbol{T}_{\boldsymbol{i}}, \boldsymbol{T}_{\boldsymbol{k}}\right)$ and bending moments $\left(\boldsymbol{M}_{\boldsymbol{i}}, \boldsymbol{M}_{\boldsymbol{k}}\right)$ in the joints $\boldsymbol{i}$ and $\boldsymbol{k}$. The convention of the positive indications of displacements and forces is presented in fig. 1.

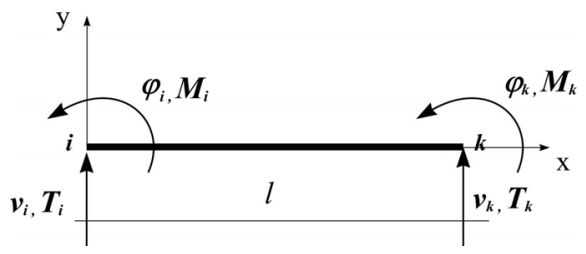

Figure 1: $\quad$ Generalized displacement and forces at the member ends.

The relation between the vector of generalized forces and the vector of generalized member displacements is:

$$
\boldsymbol{R}=\boldsymbol{k} \boldsymbol{q}-\boldsymbol{Q},
$$

where:

$$
\begin{gathered}
\boldsymbol{q}^{\mathrm{T}}=\left[\begin{array}{llll}
\boldsymbol{q}_{1} & \boldsymbol{q}_{2} & \boldsymbol{q}_{3} & \boldsymbol{q}_{4}
\end{array}\right]=\left[\begin{array}{llll}
\boldsymbol{\vartheta}_{\mathrm{i}} & \boldsymbol{\varphi}_{\mathrm{i}} & \boldsymbol{\vartheta}_{\mathrm{k}} & \boldsymbol{\varphi}_{\mathrm{k}}
\end{array}\right], \\
\boldsymbol{R}^{\mathrm{T}}=\left[\begin{array}{llll}
\boldsymbol{R}_{1} & \boldsymbol{R}_{2} & \boldsymbol{R}_{3} & \boldsymbol{R}_{4}
\end{array}\right]=\left[\begin{array}{llll}
\boldsymbol{T}_{\mathrm{i}} & \boldsymbol{M}_{\mathrm{i}} & \boldsymbol{T}_{\mathrm{k}} & \boldsymbol{M}_{\mathrm{k}}
\end{array}\right], \\
\boldsymbol{Q}^{\mathrm{T}}=\left[\begin{array}{llll}
\boldsymbol{Q}_{1} & \boldsymbol{Q}_{2} & \boldsymbol{Q}_{3} & \boldsymbol{Q}_{4}
\end{array}\right]=\left[\begin{array}{llll}
\boldsymbol{T}_{\boldsymbol{i}} & \boldsymbol{M}_{\boldsymbol{i}} & \boldsymbol{T}_{\boldsymbol{k}} & \boldsymbol{M}_{\boldsymbol{k}}
\end{array}\right], \\
\boldsymbol{k}=\left[\begin{array}{lllll}
\boldsymbol{k}_{11} & \boldsymbol{k}_{12} & \boldsymbol{k}_{13} & \boldsymbol{k}_{14} \\
\boldsymbol{k}_{21} & \boldsymbol{k}_{22} & \boldsymbol{k}_{23} & \boldsymbol{k}_{24} \\
\boldsymbol{k}_{31} & \boldsymbol{k}_{32} & \boldsymbol{k}_{33} & \boldsymbol{k}_{34} \\
\boldsymbol{k}_{41} & \boldsymbol{k}_{42} & \boldsymbol{k}_{43} & \boldsymbol{k}_{44}
\end{array}\right],
\end{gathered}
$$

are the generalized displacement vector, the generalized force vector, the equivalent loading vector and the member stiffness matrix, respectively.

Besides direct determination of the stiffness matrix and the equivalent load vector based on the clear geometric and physical meaning of its elements, very often variational procedure for deriving, based on stationariness of the function of member potential energy is used in matrix analysis.

In the case of a straight member bending in plane, the relationship between displacement $\boldsymbol{v}(\boldsymbol{x})$ of whichever point of a member axis and the parameter of displacements at the member ends can be the most easily obtained starting from the homogeneous differential equation of bending:

$$
\mathrm{EI} \frac{d^{4} v(x)}{d x^{4}}=0,
$$

whose solution can be written as a polynomial of the third order, which follows:

$$
v(x)=\alpha_{1}+\alpha_{2} x+\alpha_{3} x^{2}+\alpha_{4} x^{3} .
$$


Coefficients $\alpha_{i}(i=1,2,3,4)$ are defined from boundary conditions at the member ends. Interpolation functions in the shape of Hermit's polynomials determined for a fixed-end member are given in Sekulovic [3]. In the case of a member with semi-rigid connections at the $i$ and $k$ ends, the interpolation functions (2) can be derived from differential equation (1) and the boundary conditions.

When unit translation $\mathrm{q}_{1}=1$ is applied to the joint $i$ of a member, while all other generalized displacements are equal to zero, what follows can be written according to fig. 1 .

$$
\alpha_{i k}^{*}=\left[\mu_{i k}-\left(1-\mu_{i k}\right) \mu_{k i} \frac{b_{i k}}{a_{i k}}\right] \frac{1}{l} ; \quad \alpha_{k i}^{*}=\left[\mu_{k i}-\left(1-\mu_{k i}\right) \mu_{i k} \frac{b_{i k}}{a_{k i}}\right] \frac{1}{l}
$$

where $\mu_{\mathrm{ik}}$ and $\mu_{\mathrm{ki}}$ are the rigidity level of joint connections at the ends of a member, which can be determined numerically or experimentally.
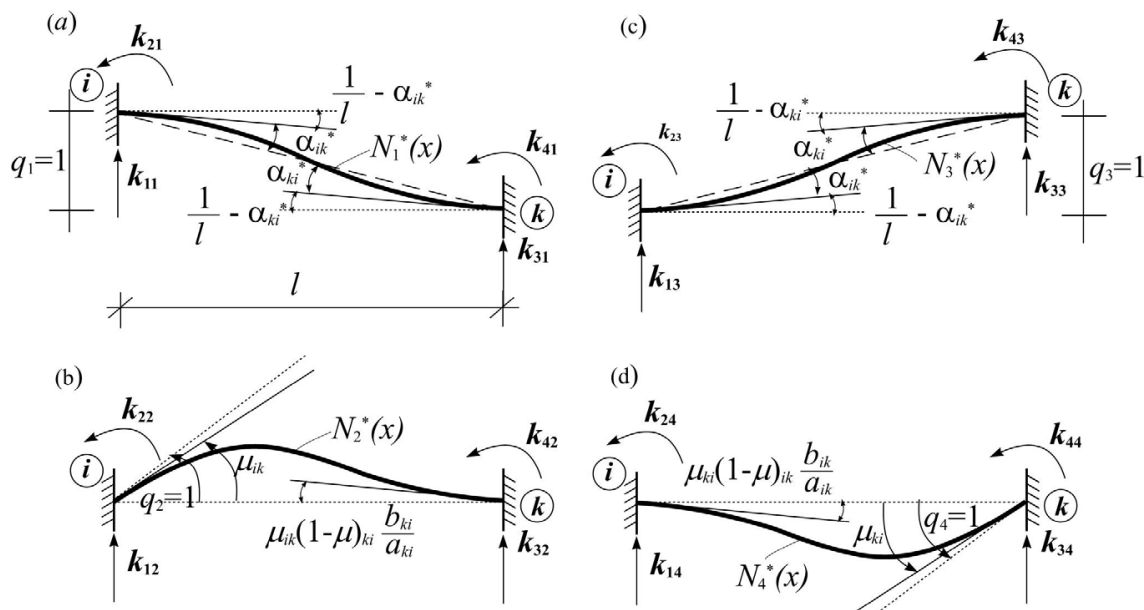

(d)

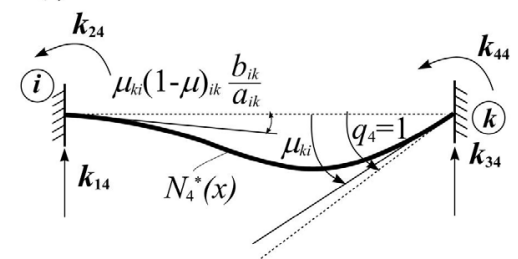

Figure 2: $\quad$ Physical meaning of the elements of stiffness matrix.

Boundary conditions for semi-rigid connections at the ends $\boldsymbol{i}$ and $\boldsymbol{k}$ of a member $i k$, from which coefficients $\alpha_{i}(i=1,2,3,4)$ appearing in expression (4) can be determined, are written in the following form:

$$
x=0\left\{\begin{array}{c}
v(x)=v_{i}=\alpha_{1}=1 \\
\varphi(x)=\varphi_{i}=\alpha_{2}=-\left(\frac{1}{l}-\alpha_{i k}^{*}\right)
\end{array} \quad x=l\left\{\begin{array}{c}
v(x)=v_{k}=1+\alpha_{2} l+\alpha_{3} l^{2}+\alpha_{4} l^{3}=0 \\
\varphi(x)=\varphi_{k}=\alpha_{2}+2 \alpha_{3} l+3 \alpha_{4} l^{2}=-\left(\frac{1}{l}-\alpha_{k i}^{*}\right)
\end{array}\right.\right.
$$

Then, according to (4), the elements of the interpolation function matrix, when unit displacement $\mathrm{q}_{1}=1$ of the joint $\boldsymbol{i}$, unit rotation $\mathrm{q}_{2}=1$ at the end $\boldsymbol{i}$, unit displacement $\mathrm{q}_{3}=1$ of the joint $\boldsymbol{k}$ and unit rotation $\mathrm{q}_{4}=1$ of the joint $\boldsymbol{k}$ are applied separately, while all of the other generalized displacements are equal to zero, interpolation functions $\boldsymbol{N}_{1}^{*}, \boldsymbol{N}_{2}^{*}, \boldsymbol{N}_{3}$ and $\boldsymbol{N}_{4}^{*}$, respectively, can be determined.

Interpolation functions $N_{\mathrm{m}}^{*}(\mathrm{~m}=1, . ., 4)$ represent Hermit's polynomials of the first order and their diagrams are shown in fig. 2. In the limit cases when a 
member is rigidly connected at its ends $\boldsymbol{i}$ and $\boldsymbol{k}$ (rigidly fixed-end member), that is $\mu_{\boldsymbol{i k}}=\mu_{\boldsymbol{k} \boldsymbol{i}}=1$, expressions (8) have already known values (Sekulovic [3]).

So, the matrix of interpolation functions can be shown in the following form:

$$
N^{*}=\left[\begin{array}{llll}
N_{1}^{*}(x) & N_{2}^{*}(x) & N_{3}^{*}(x) & N_{4}^{*}(x)
\end{array}\right],
$$

where

$$
\begin{aligned}
& N_{1}^{*}(x)=v(x)_{q_{1}=1}=1-\left(\frac{1}{l}-\alpha_{i k}^{*}\right) x-\frac{2 \alpha_{i k}^{*}+\alpha_{k i}^{*}}{l} x^{2}+\frac{\alpha_{i k}^{*}+\alpha_{k i}^{*}}{l^{2}} x^{3}, \\
& N_{2}^{*}(x)=\mu_{i k} x-\frac{2 \mu_{i k}-\mu_{k i}+\alpha_{k i}^{*} l}{l} x^{2}+\frac{\mu_{i k}-\mu_{k i}+\alpha_{k i}^{*} l}{l^{2}} x^{3}, \\
& N_{3}^{*}(x)=\left(\frac{1}{l}-\alpha_{i k}^{*}\right) x+\frac{2 \alpha_{i k}^{*}+\alpha_{k i}^{*}}{l} x^{2}-\frac{\alpha_{i k}^{*}+\alpha_{k i}^{*}}{l^{2}} x^{3}, \\
& N_{4}^{*}(x)=\left(\mu_{i k}-\alpha_{i k}^{*} l\right) x-\frac{2 \mu_{i k}+\mu_{k i}-2 \alpha_{k i}^{*} l}{l} x^{2}+\frac{\mu_{i k}+\mu_{k i}-\alpha_{k i}^{*} l}{l^{2}} x^{3} .
\end{aligned}
$$

Interpolation function $\boldsymbol{N}_{\mathrm{m}}^{*}(x)$ represents an elastic line of a semi-rigidly fixed-end member due to generalized displacement $\mathrm{q}_{\mathrm{m}}=1(\mathrm{~m}=1,2,3,4)$, while all of the other generalized displacements are $\mathrm{q}_{\mathrm{n}}=0, \mathrm{n} \neq \mathrm{m}$.

\subsection{Stiffness matrix of a semi-rigidly fixed-end member}

The stiffness matrix of a semi-rigidly connected member is obtained after the second derivatives of the interpolation functions have been determined and is as follows:

$$
k^{*}=\operatorname{EI} \int_{0}^{\ell}\left\{\begin{array}{l}
N_{1}^{* \prime}(x) \\
N_{2}^{* \prime \prime}(x) \\
N_{3}^{* \prime \prime}(x) \\
N_{4}^{* \prime \prime}(x)
\end{array}\right\}\left[\begin{array}{lllll}
N_{1}^{* \prime \prime}(x) & N_{2}^{*}(x) & N_{3}^{*}(x) & N_{4}^{*}(x)
\end{array}\right] \mathrm{d} x,
$$

where:

$$
\begin{aligned}
& \boldsymbol{k}_{11}^{*}=\frac{4 \mathrm{EI}}{\ell}\left[\alpha_{i k}^{* 2}+\alpha_{i k}^{*} \alpha_{k i}^{*}+\alpha_{k i}^{* 2}\right], \\
& \boldsymbol{k}_{12}^{*}=\frac{2 \mathrm{EI}}{\ell}\left[2\left(\alpha_{i k}^{*} \mu_{i k}+\alpha_{k i}^{* 2} \ell-\alpha_{k i}^{*} \mu_{k i}\right)-\alpha_{i k}^{*} \mu_{k i}+\alpha_{i k}^{*} \alpha_{k i}^{*} \ell+\alpha_{k i}^{*} \mu_{i k}\right], \\
& \boldsymbol{k}_{13}^{*}=-\frac{4 \mathrm{EI}}{\ell}\left[\alpha_{i k}^{* 2}+\alpha_{i k}^{*} \alpha_{k i}^{*}+\alpha_{k i}^{* 2}\right]=-k_{11}^{*},
\end{aligned}
$$




$$
\begin{aligned}
& \boldsymbol{k}_{14}^{*}=\frac{2 \mathrm{EI}}{\ell}\left[2\left(\alpha_{i k}^{*} \mu_{i k}-\alpha_{k i}^{* 2} \ell+\alpha_{k i}^{*} \mu_{k i}\right)+\alpha_{i k}^{*} \mu_{k i}-\alpha_{i k}^{*} \alpha_{k i}^{*} \ell+\alpha_{k i}^{*} \mu_{i k}\right], \\
& \boldsymbol{k}_{22}^{*}=\frac{4 \mathrm{EI}}{\ell}\left[\mu_{\boldsymbol{i k}}^{2}-\mu_{\boldsymbol{i} \boldsymbol{k}} \mu_{\boldsymbol{k} \boldsymbol{i}}+\mu_{\boldsymbol{k} \boldsymbol{i}}^{2}+\boldsymbol{\alpha}_{\boldsymbol{k} \boldsymbol{i}}^{*} \mu_{\boldsymbol{i} \boldsymbol{k}}^{\ell}-2 \alpha_{\boldsymbol{k} \boldsymbol{i}}^{*} \mu_{\boldsymbol{k} \boldsymbol{i}} \ell+\alpha_{\boldsymbol{k} \boldsymbol{i}}^{* 2} \ell^{2}\right] \text {, } \\
& \boldsymbol{k}_{23}^{*}=-\frac{2 \mathrm{EI}}{\ell}\left[2\left(\alpha_{i k}^{*} \mu_{i k}+\alpha_{k i}^{* 2} \ell-\alpha_{k i}^{*} \mu_{k i}\right)-\alpha_{i k}^{*} \mu_{k i}+\alpha_{i k}^{*} \alpha_{k i}^{*} \ell+\alpha_{k i}^{*} \mu_{i k}\right]=-k_{12}^{*}, \\
& \boldsymbol{k}_{24}^{*}=\frac{2 \mathrm{EI}}{\ell}\left[2\left(\mu_{i k}^{2}-\alpha_{i k}^{*} \mu_{i k} \ell-\mu_{k i}^{2}+\alpha_{k i}^{*} \mu_{k i} \ell\right)+\alpha_{i k}^{*} \mu_{k i} \ell+\alpha_{k i}^{*} \mu_{i k} \ell-\alpha_{i k}^{*} \alpha_{k i}^{*} \ell^{2}\right] \text {, } \\
& \boldsymbol{k}_{33}^{*}=\frac{4 \mathrm{EI}}{\ell}\left[\alpha_{i k}^{* 2}+\alpha_{i k}^{*} \alpha_{k i}^{*}+\alpha_{k i}^{* 2}\right]=k_{11}^{*}, \\
& \boldsymbol{k}_{34}^{*}=-\frac{2 \mathrm{EI}}{\ell}\left[2\left(\alpha_{i k}^{*} \mu_{i k}-\alpha_{k i}^{* 2} \ell+\alpha_{k i}^{*} \mu_{k i}\right)+\alpha_{i k}^{*} \mu_{k i}-\alpha_{i k}^{*} \alpha_{k i}^{*} \ell+\alpha_{k i}^{*} \mu_{i k}\right]=-k_{14}^{*}, \\
& \boldsymbol{k}_{44}^{*}=\frac{4 \mathrm{EI}}{\ell}\left[\mu_{i \boldsymbol{k}}^{2}+\mu_{i k} \mu_{\boldsymbol{k} i}+\mu_{\boldsymbol{k} i}^{2}-2 \alpha_{i \boldsymbol{k}}^{*} \mu_{\boldsymbol{i} \boldsymbol{k}} \ell-\alpha_{\boldsymbol{i k}}^{*} \mu_{\boldsymbol{k} \boldsymbol{i}} \ell+\alpha_{i \boldsymbol{k}}^{* 2} \ell^{2}\right] \text {. }
\end{aligned}
$$

When the axial forces effect on deformation is taken into account, the stiffness matrix of a semi-rigidly fixed-end member can be written as follows:

$$
\boldsymbol{k}^{*}=\left[\begin{array}{cccccc}
\frac{\mathrm{EF}}{\ell} & 0 & 0 & \frac{\mathrm{EF}}{\ell} & 0 & 0 \\
& \boldsymbol{k}_{11}^{*} & \boldsymbol{k}_{12}^{*} & 0 & \boldsymbol{k}_{13}^{*} & \boldsymbol{k}_{14}^{*} \\
& & \boldsymbol{k}_{22}^{*} & 0 & \boldsymbol{k}_{23}^{*} & \boldsymbol{k}_{24}^{*} \\
& & & \frac{\mathrm{EF}}{\ell} & 0 & 0 \\
& & & & \boldsymbol{k}_{33}^{*} & \boldsymbol{k}_{34}^{*} \\
\operatorname{sim} . & & & & & \boldsymbol{k}_{44}^{*}
\end{array}\right] .
$$

\subsection{Vector of equivalent load}

In the matrix analysis of structures, the external action along individual members can be replaced by concentrated load at the ends of the member. In the case of bending in plane, the load vector components are presented in fig. 3 and they are equal to the negative values of reactions of elastically fixed members under given external action, which can be a load normal to the axis of the member, as well as temperature differences between temperatures on the upper and lower surfaces of the member.

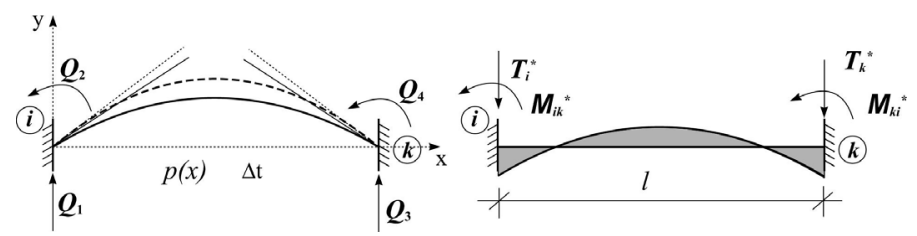

Figure 3: $\quad$ Vector of equivalent load. 


$$
\boldsymbol{Q}^{*}=\left[\begin{array}{l}
\boldsymbol{Q}_{1} \\
\boldsymbol{Q}_{2} \\
\boldsymbol{Q}_{3} \\
\boldsymbol{Q}_{4}
\end{array}\right]=-\left[\begin{array}{c}
\boldsymbol{T}_{\boldsymbol{i}}^{*} \\
\boldsymbol{M}_{\boldsymbol{i k}}^{*} \\
\boldsymbol{T}_{\boldsymbol{k}}^{*} \\
\boldsymbol{M}_{\boldsymbol{k} \boldsymbol{i}}^{*}
\end{array}\right]_{(\mathrm{o})}-\left[\begin{array}{c}
\boldsymbol{T}_{\boldsymbol{i}}^{*} \\
\boldsymbol{M}_{\boldsymbol{i} \boldsymbol{k}}^{*} \\
\boldsymbol{T}_{\boldsymbol{k}}^{*} \\
\boldsymbol{M}_{\boldsymbol{k} \boldsymbol{i}}^{*}
\end{array}\right]_{(\Delta \mathrm{t})} .
$$

The vector of equivalent load for a member with semi-rigid end connections is:

$$
Q^{T}=\int_{0}^{l} p(x) N^{*}(x) \mathrm{d} x .
$$

For example, in the case of uniform load, where $\mathrm{p}(\mathrm{x})=\mathrm{p}=\mathrm{const}$, it can be written in the following form:

$$
Q^{*}=p \int_{0}^{\ell}\left\{\begin{array}{c}
1-\left(\frac{1}{\ell}-\alpha_{i k}^{*}\right) x-\frac{2 \alpha_{i k}^{*}+\alpha_{k i}^{*}}{\ell} x^{2}+\frac{\alpha_{i k}^{*}+\alpha_{k i}^{*}}{\ell^{2}} x^{3} \\
\mu_{i k} x-\frac{2 \mu_{i k}-\mu_{k i}+\alpha_{k i}^{*} \ell}{\ell} x^{2}+\frac{\mu_{i k}-\mu_{k i}+\alpha_{k i}^{*} \ell}{\ell^{2}} x^{3} \\
\left(\frac{1}{\ell}-\alpha_{i k}^{*}\right) x+\frac{2 \alpha_{i k}^{*}+\alpha_{k i}^{*}}{\ell} x^{2}-\frac{\alpha_{i k}^{*}+\alpha_{k i}^{*}}{\ell^{2}} x^{3} \\
\left(\mu_{i k}-\alpha_{i k}^{*} \ell\right) x-\frac{2 \mu_{i k}+\mu_{k i}-2 \alpha_{k i}^{*} \ell}{\ell} x^{2}+\frac{\mu_{i k}+\mu_{k i}-\alpha_{k i}^{*} \ell}{\ell^{2}} x^{3}
\end{array}\right\}
$$

After integration it becomes:

$$
Q^{*}=\frac{p \ell^{2}}{12}\left\{\begin{array}{c}
\frac{6}{\ell}+\alpha_{i k}^{*}-\alpha_{k i}^{*} \\
\mu_{i k}+\mu_{k i}-\alpha_{k i}^{*} \ell \\
\frac{6}{\ell}-\alpha_{i k}^{*}+\alpha_{k i}^{*} \\
\mu_{i k}-\mu_{k i}-\alpha_{i k}^{*} \ell
\end{array}\right\}, \quad \lim _{\substack{\mu_{i k} \rightarrow 1 \\
\mu_{k i} \rightarrow 1}} Q^{*}=\frac{p}{2}\left\{\begin{array}{c}
1 \\
\frac{\ell}{6} \\
1 \\
-\frac{\ell}{6}
\end{array}\right\}, \underset{\substack{\mu_{i k} \rightarrow 1 \\
\mu_{k i} \rightarrow 0}}{\lim } Q^{*}=\frac{p}{2}\left\{\begin{array}{c}
\frac{5}{4} \\
\frac{\ell}{4} \\
\frac{3}{4} \\
0
\end{array}\right\} \cdot
$$

For other cases of loading, such as linear distributed load and concentrated force, concentrated moment vectors of equivalent loading are also derived (Zlatkov [4]).

In the case of the influence of constant temperature differences along the member axis the equivalent load vector is obtained as follows: 


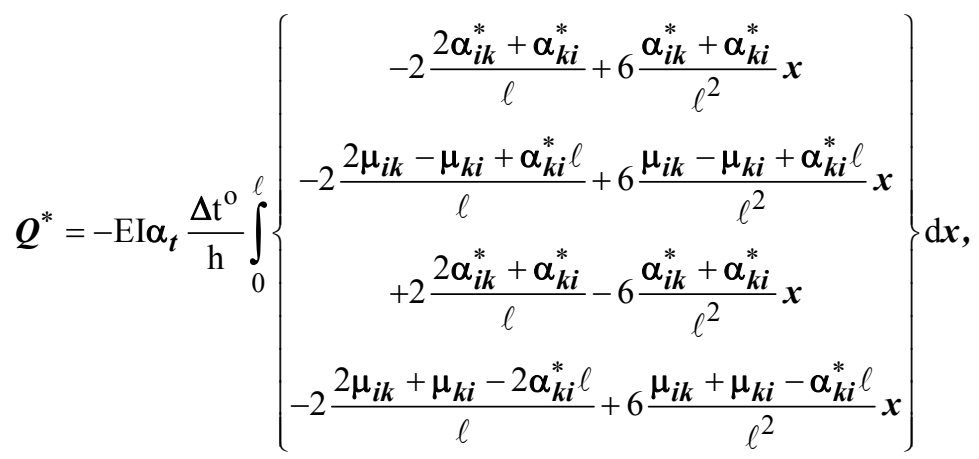

and after integration it is:

$$
\begin{aligned}
& Q^{*}=-\mathrm{EI}_{\mathrm{t}} \frac{\Delta \mathrm{t}^{\mathrm{o}}}{\mathrm{h}}\left\{\begin{array}{c}
-\alpha_{\boldsymbol{i k}}^{*}+\alpha_{\boldsymbol{k} i}^{*} \\
-\mu_{\boldsymbol{i k}}-\mu_{\boldsymbol{k} i}+\alpha_{\boldsymbol{k} i}^{*} \ell \\
\alpha_{i k}^{*}-\alpha_{\boldsymbol{k} i}^{*} \\
-\mu_{\boldsymbol{i k}}+\mu_{\boldsymbol{k} i}+\alpha_{\boldsymbol{k} i}^{*} \ell
\end{array}\right\}, \\
& \lim _{\substack{\mu_{i k} \rightarrow 1 \\
\mu_{k i} \rightarrow 1}} Q^{*}=-\mathrm{EI} \alpha_{\mathrm{t}} \frac{\Delta \mathrm{t}^{\mathrm{o}}}{\mathrm{h}}\left\{\begin{array}{c}
0 \\
-1 \\
0 \\
1
\end{array}\right\}, \lim _{\substack{\mu_{i k} \rightarrow 1 \\
\mu_{k i} \rightarrow 0}} Q^{*}=-\mathrm{EI} \alpha_{\mathrm{t}} \frac{\Delta \mathrm{t}^{\mathrm{o}}}{\mathrm{h}}\left\{\begin{array}{c}
-\frac{3}{2 \ell} \\
-\frac{3}{2} \\
\frac{3}{2 \ell} \\
0
\end{array}\right\} \text {. }
\end{aligned}
$$

\section{Some results of the static design of a system with semi-rigid connections}

The derived expressions for the stiffness matrix and equivalent load vectors can be incorporated in available software in order to enable structural design of linear systems with semi-rigid connections. In this paper the well-known computer program STRESS, intended for the linear elastic analysis of plane or space structures, is applied to illustrate the above presented theoretical approach.

The design of structures with semi-rigid connections using STRESS differs from the standard procedure only in commands containing data about members, i.e. it is necessary to form the stiffness matrices of members. Instead of giving cross-section characteristics of prismatic members (area and moment of inertia for the main axes) through the command MEMBER PROPERTIES PRISMATIC, properties of a member are described through the command STIFFNESS GIVEN, in which case the basic stiffness matrix elements for semirigidly connected members are input directly. 
Load cannot be included by the command MEMBER LOADS as load distributed along the member length, but has to be represented as equivalent end load and input by the command MEMBER END LOADS in the following form:

M START FORCE X $\alpha_{1} \quad \mathrm{Y} \quad \alpha_{2} \quad \mathrm{Z} \alpha_{3}$ MOMENT X $\alpha_{4} \mathrm{Y} \quad \alpha_{5} \mathrm{Z} \quad \alpha_{6}$

M END FORCE X $\alpha_{1}$ Y $\alpha_{2} \quad Z \alpha_{3}$ MOMENT X $\alpha_{4} \mathrm{Y} \alpha_{5} \mathrm{Z} \alpha_{6}$, where $\mathrm{M}$ is the notation of a member, FORCE $\mathrm{X}, \mathrm{Y}, \mathrm{Z}$ are the end forces in directions $\mathrm{x}, \mathrm{y}, \mathrm{Z}$ with their numerical values $\alpha_{1}, \alpha_{2}, \alpha_{3}$ and MOMENT X,Y,Z are the end moments in directions $\mathrm{x}, \mathrm{y}, \mathrm{z}$ with their numerical values $\alpha_{4}, \alpha_{5}, \alpha_{6}$.

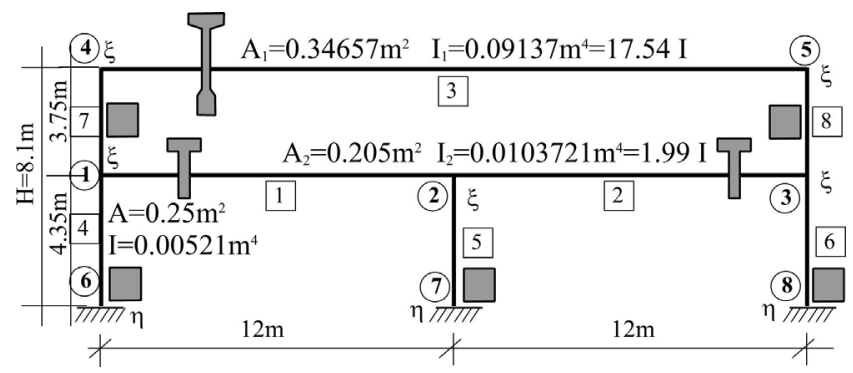

Figure 4: Static scheme of a RC frame in the AMONT prefabricated structural system.

The real frame structure, under real loading (presented in fig. 4), is considered as an illustration of this application to frames with semi-rigid connections. This is a two-floor RC frame of the AMONT prefabricated structural system, Morava Krusce, Serbia, with a span of $24 \mathrm{~m}$, column cross section of $50 \times 50 \mathrm{~cm}$ and beam cross sections as shown at fig. 4. From the results of tests of the AMONT connections, Ristic [5], it has been found that the connection column to foundation is almost absolutely rigid and because of that the level of rigidity is adopted as $\mu_{61}=\mu_{72}=\mu_{83}=\eta=1$ (fixed-end member) in the numerical example, while the connection beam to column behaves as $75 \%$ fixed, so it is adopted as $\mu_{12}=\mu_{21}=\mu_{23}=\mu_{32}=\mu_{45}=\mu_{54}=\mu_{41}=\mu_{53}=\mu_{27}=\xi=0.75$.

The computer program MKS2 is composed for the calculation of the elements of the base stiffness matrix for various combinations of the level of rigidity of connections at the member ends according to (10) (Zlatkov [4]). The stiffness sub matrices required as input data in STRESS (Stankovic [6]) for all members of the system (shown in fig. 4) are:

$$
\begin{aligned}
& \boldsymbol{k}_{1}^{*}=\boldsymbol{k}_{2}^{*}=\left[\begin{array}{ccc}
580833.3 & 0 & 0 \\
0 & 1606.5 & -9639.4 \\
0 & -9639.4 & 82547.2
\end{array}\right], \boldsymbol{k}_{3}^{*}=\left[\begin{array}{ccc}
490974.2 & 0 & 0 \\
0 & 1770.2 & -21242.1 \\
0 & -21242.1 & 364022.7
\end{array}\right] \text {, } \\
& \boldsymbol{k}_{4}^{*}=\boldsymbol{k}_{6}^{*}=\left[\begin{array}{ccc}
195403 . & 0 & 0 \\
0 & 25837.3 & -56196.1 \\
0 & -56196.1 & 162968.8
\end{array}\right], \boldsymbol{k}_{5}^{*}=\left[\begin{array}{ccc}
195403 . & 0 & 0 \\
0 & 20997.5 & -42147.1 \\
0 & -42147.1 & 122226.6
\end{array}\right] \text {, }
\end{aligned}
$$




$$
\boldsymbol{k}_{7}^{*}=\boldsymbol{k}_{8}^{*}=\left[\begin{array}{ccc}
2266666.7 & 0 & 0 \\
0 & 32751.4 & -56684.8 \\
0 & -56684.8 & 141712 .
\end{array}\right] \text {. }
$$

The equivalent load vector for members 1,2 is:

$$
\begin{aligned}
& \boldsymbol{Q}_{12}^{*}=\boldsymbol{Q}_{21}^{*}=\boldsymbol{Q}_{23}^{*}=\boldsymbol{Q}_{32}^{*}=330.93 \mathrm{kN}, \\
& \boldsymbol{M}_{12}^{*}=-\boldsymbol{M}_{21}^{*}=\boldsymbol{M}_{23}^{*}=-\boldsymbol{M}_{32}^{*}=-558.444 \mathrm{kNm},
\end{aligned}
$$

and the equivalent load vector for member 3 is:

$$
\boldsymbol{Q}_{45}^{*}=\boldsymbol{Q}_{54}^{*}=277.92 \mathrm{kN}, \quad \boldsymbol{M}_{45}^{*}=-\boldsymbol{M}_{54}^{*}=-937.98 \mathrm{kNm} .
$$

A diagram of bending moments is shown in fig. 5 for the adopted rigidity level of connections for the chosen AMONT frame. The values of bending moments in the case of absolutely fixed members in joints are shown in brackets. The differences between these two models are evident and they point out the fact that the calculation allowing for the rigidity of connections has is justified because it is closer to the real behaviour of the structure.

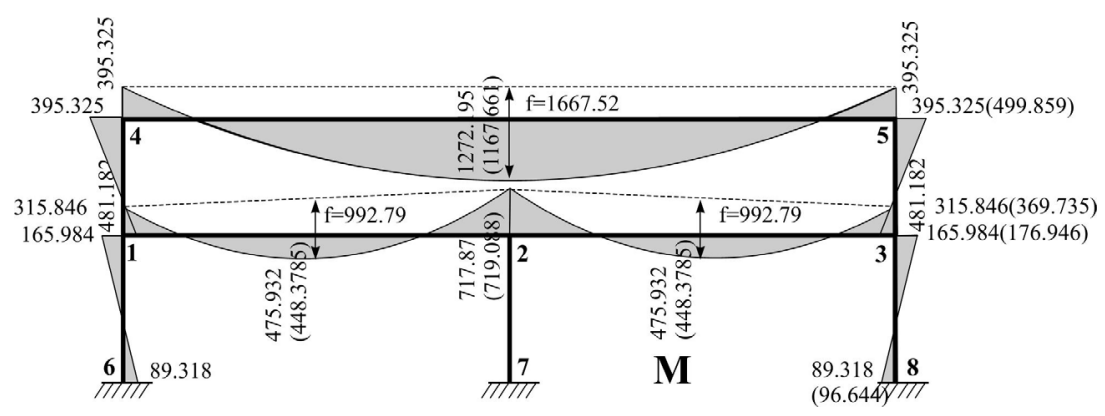

Figure 5: Diagram of bending moments for $\eta=1.0$ and $\xi=0.75$ (in brackets are values for $\xi=\eta=1.0$ ).

\section{Conclusion}

In this paper the stiffness matrix and the vector of equivalent load for systems with semi-rigid connections are derived using the variation procedure on the basis of the deformation method. These terms can be incorporated in the available software in order to enable structural design of linear systems with semi-rigid connections. In this paper the well-known computer program STRESS, intended for the linear elastic analysis of plane or space structures, is applied to illustrate the presented theoretical approach.

The numerical example of the model with semi-rigid connections, which represents the real frame structure under real loading, is considered. Comparing the obtained static and deformation values with those for the model with standard connections, significant differences are observed, meaning that care has 
to be taken in practical civil engineering design, particularly for the case of precast RC systems.

The presented procedure enables a designer to choose a model with different levels of rigidity of each member end in one joint, which can be determined experimentally, and to find out what the redistribution of the internal influences looks like depending on the real achieved level of rigidity of connections. This is particularly useful in the case of a very important structure, which can be tested after construction with the aim of finding out the real behaviour of the structure among all the levels of rigidity of its connections. Then the results of the tests can be used for designing a more realistic model that could be used in further analysis in the case of accidental loading, such as seismic forces, or in the case of changing of the purpose of the construction, when it is economically justified.

\section{Acknowledgement}

This research is supported by the Ministry of Science of the Republic of Serbia, within the framework of the project Experimental and Theoretical Research of Real Connections at Reinforced Concrete and Composite Structures under Static and Dynamic Loading, No 16001, for the period 2008-2010.

\section{References}

[1] Djuric, M., Jovanovic, P., Teorija okvirnih konstrukcija, Grdjevinska knjiga, Beograd, 1972.

[2] Milicevic, M., Zdravkovic, S., Uticaj stepena krutosti veze na velicinu kriticnog opterecenja i promenu naprezanja u linijskim sistemima, Simpozijum Nova tehnicka regulativa vo gradeznoto konstrukterstvo, pp. D8-1-D8-7, Skopje, 1986.

[3] Sekulovic, M., Matrix analysis of structure, Gradjevinska knjiga, Beograd, 1991.

[4] Zlatkov, D., Analiza linijskih sistema sa polukrutim vezama stapova u cvorovima, 1-233, Gradjevinski fakultet Nis, 1998.

[5] Ristic, D., Micov, V., Zisi, N., Dimitrovski, T., Attesting of Static and Dynamic Stability of the Typified Modulus of a Hall Program of the Precast RC Structural System AMONT-Krusce IZIIS Skopje, 1998.

[6] Stankovic, S., Djordjevic, D.J., Stress, Programski sistem za staticki proracun inzenjerskih konstrukcija-uputstvo za koriscenje programa, Trion Computers, Nis, 1991.

[7] Milicevic, M., Zdravkovic, S., Zlatkov, D., Matrix analysis of systems with semi-rigid connections of members, Proceeding of $21^{\text {th }}$ Yugoslav congress of theoretical and applied mechanics, pp. 265-268, Nis, 1995.

[8] Milicevic, M., Zdravkovic, S., Zlatkov, D., Kostadinov, B., Matrix Formulation of Design and Testing of Structures with Semi-Rigid Connections, Structural Engineers World Congress, pp. 266, San Francisco, California, 1998. 